\title{
On the MIMO OTA test system
}

\author{
Jukka-Pekka Nuutinen ${ }^{1}$, Pekka Kyösti ${ }^{1}, Y$ u Gao ${ }^{1}$, Michael D. Foegelle ${ }^{2}$ \\ ${ }^{1}$ Elektrobit System Test \\ Tutkijantie 7, FIN-90570 Finland \\ Jukka-pekka.nuutinen@elektrobit.com \\ ${ }^{2}$ ETS-Lindgren \\ 1301 Arrow Point Drive, Cedar Park, TX 78613 \\ Michael.foegelle@ets-lindgren.com
}

\begin{abstract}
This paper discusses the radio channel modeling for MIMO Over-The-Air testing (OTA). The MIMO OTA is needed for the small devices testing, utilizing multiple antennas. The technologies that utilize multiple antennas are emerging technologies, such as LTE-A and WiMAX evolution (IEEE802.11m), but we also have seen tremendeous increase of MIMO in WLAN type of applications, like IEEE802.11n. This paper discusses also about the testing challenge that MIMO poses for realistic testing.

Index Terms - MIMO OTA, radio channel modeling
\end{abstract}

\section{INTRODUCTION}

In SISO (Single Input Single Output) systems, the dominant radio channel multi-path effects limiting the performance, were delay spread and Doppler spread. The delay spread, caused by large delay echoes of the signals is typically causing intersymbol interferences, whereas Doppler spread, caused by terminal movement is typically causing intercarrier interference. Thus we may say that the environment and the speed of the terminal typically degrades the SISO system performance. Neither of these are antenna dependent. Thus it was rather clear that good picture about the performance could be obtained by omitting the antenna effects and that justified the conducted testing.

As the MIMO technologies are now introduced in various standards, such as HSPA (High Speed Packet Access), LTE (Long Term Evolution), IEEE802.16e (mobile WiMAX) and its evolution IEEE802.16m (gigabit's WiMAX) as well as all the IMT-A proposals, we have to look the situation from a bit different perspective. Namely, the idea of using MIMO is to increase the spectral efficiency, e.g. reusing the same frequency band through uncorrelated channels. Thus 1) the radio channel models incorporate the spatial domain and 2) the correlation defines the MIMO efficiency. The correlation and spatial domain propagation both are antenna dependent. This means in practice, that no matter how clever the MIMO algorithm is, if the propagation media do not support uncorrelated channels, the MIMO performance is degraded. Also the antenna design (especially mutual coupling or leakage from the antenna branches) affects a lot to the correlation properties. Thus, we need to introduce to the test system, besides the spatial domain propagation, also incorporate antenna effects. The most realistic way to include antenna effects is to use them as they would be in a final product. This leads to the wireless test system that is introduced in figure 1.

\section{MIMO OTA TEST SYSTEM}

MIMO OTA stands for the Multiple Input Multiple Output Over-The-Air test system (figure 1). The method is that it is meant for testing of relatively small MIMO devices and testing is done in wireless manner (i.e. over the air). Originally, the topic has been discussed in various papers, including [1]. MIMO OTA enables us to use true, end user equipment as a Device Under Test (DUT), thus giving full understanding of the performance of the DUT as well as compare DUTs in equivalent conditions.

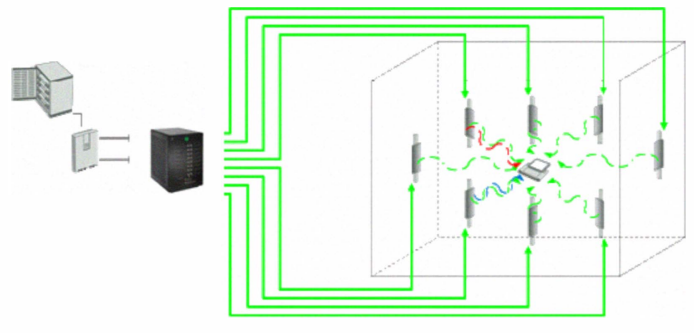

Fig 1. The MIMO OTA concept.

The essential components of MIMO OTA test system are 1) test transmitter (e.g. a communication tester or a base station emulator), 2) fading emulator with MIMO OTA channel modeling tool, 3) anechoic chamber, 4) OTA antennas and 5) Device under test (DUT). The DUT is typically relatively small device. DUT is typically UE, terminal or laptop device.

The spatial domain propagation is generated by a number of probe antennas in the chamber. Even though, the number of antennas is limited to the number of the available channels, the MIMO OTA system is able to generate by weighting any kind of angular behavior into chamber. The correlation is a consequence of this angular propagation behavior and antenna design. Since the model has to be realistic, it has to be based on the observations, thus physical measurements. Therefore only Geometry Based Stochastic Models, such as SCM, SCME, WINNER or IMT-A models are applicable.

The most important issue is to understand the fact that the spatial correlation, defined by equation (1),

$$
\rho(D)=\frac{1}{K} \sum_{k=1}^{K} \exp \left(-j 2 \pi D \sin \left(\theta_{0}+\Delta \theta_{k}\right)\right)
$$


is dependent on the angular behavior (mean and spread). This means that the effectiveness of the MIMO is dependent on the angular characteristics of the radio channel. Thus the models we use for the MIMO OTA must be geometry based stochastic models.

\section{SIZE OF DUT}

The most critical parameter in the test system is the area that can be covered by the discrete OTA antennas. This is called quiet zone. Quiet zone is determined by the number of the probe (OTA) antennas. More we have probe antennas, bigger is the quiet zone. As a metric, we use spatial correlation. Theoretically, it should follow Bessel function of the 1st kind. Following figure depict the effects of changing the number of the OTA antennas.

\begin{tabular}{|l|l|l|}
\hline \#OTA & 1 dim autocorrelation & 2 dim autocorrelation \\
\hline 4 & &
\end{tabular}

Fig. 2. One and two dimensional autocorrelations vs. number of OTA antennas.

From figure 2 we can observe that the minimum requirement to have adequate quiet zone for handheld testing is 8 OTA antennas (in $2 \mathrm{GHz}$ the quiet zone is around $0.7 \lambda$ and thus the size of the DUT can be $12.5 \mathrm{~cm}$ ) and for lap top testing we need 16 OTA antennas (in $2 \mathrm{GHz}$ the quiet zone is around $2 \lambda$ and thus the size of the DUT can be $30 \mathrm{~cm}$ ).

\section{STANDARDIZATION OF MIMO OTA TEST S}

There are basically two major organizations that are working towards MIMO OTA standard, 3GPP and CTIA.

In 3GPP RAN 4 the situation is that the MIMO OTA is in study item phase and one potential candidate is shown in figure 1. In CTIA the work is also ongoing. In CTIA the MIMO OTA work is subdivided to the anechoic chamber group work (MACSG) and to reverberation chamber group work (reverb). It is expected that the study item will be finished in 3GPP around H2/2010 and the CTIA is a bit faster.

The COST2100 shall provide technical content to 3GPP. COST2100 is European research forum, where various Pervasive Mobile \& Ambient Wireless Communications issues are discussed. In COST2100 the SWG2.2 Compact Antenna Systems for Terminals is developing the test methods for multi-antenna devices. Documents [2] - [7] and [9] - [12] describe the recent progress of the work in COST2100 related to this MIMO OTA system.

\section{Channel MOdeling FOR MIMO OTA}

The radio channel characteristics that are essential are described by joint spatio-temporal models. They are such models that tie environment to the model in geometrical basis. An illustration of this is depicted in figure 3 .

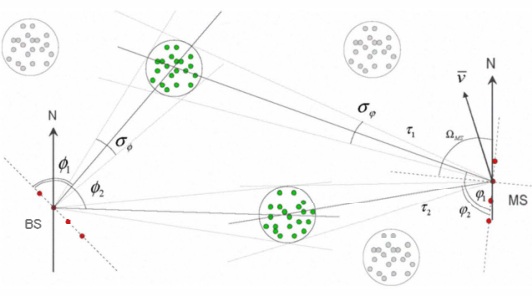

Figure 3. A geometrical radio channel model

In this kind of models, the scatterers are characterized by clusters. Clusters are characterized by mean and spread of each radio channel parameter (AoA, AoD, delay, Doppler). The mentioned parameters are different in each environment, e.g. in suburban model we have different values of the parameters than in urban environment. Examples of such models are 3GPP spatial channel model SCM, its extension SCME and WINNER channel models [14], [15].As seen from the figure 2, a nice feature of these spatio-temporal models is that they define angular behavior, thus it allows the mapping of angles to antennas in MIMO OTA system

The virtue of this is that in practice any kind of model having angular behaviour, is usable for the MIMO OTA. Model can be e.g. based on the measurement. Also, it is worth of noticing that AoA behaviour is modeled by the OTA antennas, i.e. AoD is as it would be in the original model.

The intelligence of the MIMO OTA is in above mentioned mapping from the original model to the environment in the lab. The mapping is done such that we are able to optimize the angle, correlation or both in the DUT. In principle, the mapping process is following. 


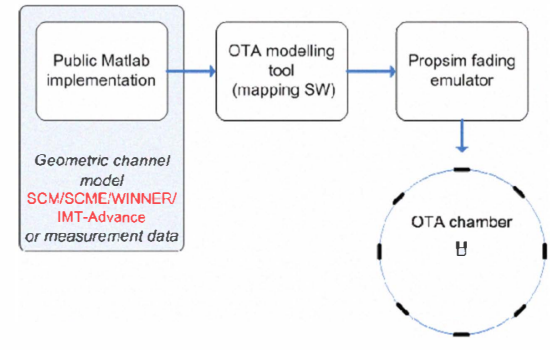

Figure 4. Generation of the OTA models.

The figure 4 says in principle that the original model is converted with optimization algorithm to the OTA model that is used in a fading emulator. As each model has several clusters with different AoA characteristics, we need e.g. 8 channels, as depicted below.
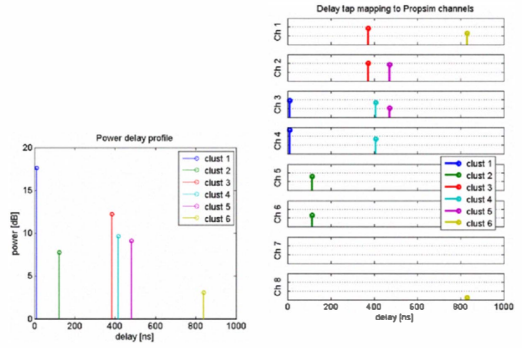

Figure 5. The original model (on left) and OTA tool mapped model (on right).

Power weights for OTA antennas are optimized to create the target spatial correlation function in the "quiet zone".

\section{VALIDATION OF RADIO CHANNEL CHARARCETISTICS}

The validation of radio channel characteristics has been done using the Propsound CS multidimensional radio channel sounder [16]. The idea of the validation is to prove that the radio channel characterics that we generate with fading emulator, can be measured in the MIMO OTA chamber. The following figure depicts the system validation.

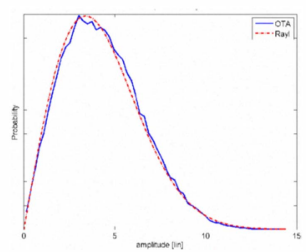

a)

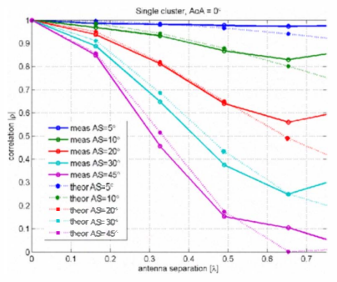

c)

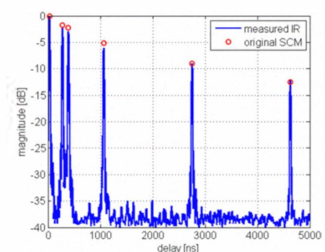

b)

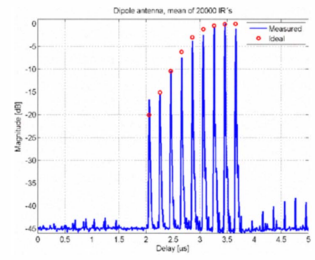

d)
Figure 6. Examples of measured validation results, a) fading distribution, b) PDP, c) correlation and d) polarization.
The figure 6 can be interpreted such that the investigated characteristics of radio channel are well preserved in the MIMO OTA test set-up.

\section{Practical example}

This section discusses about a practical performance measurement. The MIMO device under test was Linksys IEEE $802.11 \mathrm{n}$ device with $2 \times 2$ MIMO. It is a commercially available off the shelf device, illustrating that the test system can be used also with final products to compare various devices under identical radio channel conditions. The device was operated in the $2.4 \mathrm{GHz}$ band using $20 \mathrm{MHz}$ channel bandwidth. Another matching device from the same product family was used as the test equipment making up the other side of the link outside the chamber to ensure compatibility with all modes of MIMO operation of the DUT. The "downlink" signal from the tester to the DUT was routed through the channel emulator then through eight $\sim 30 \mathrm{~dB}$ power amplifiers to a circular array of eight vertically polarized broadband measurement antennas. The "uplink" from the DUT was captured using a circularly polarized antenna and fed through circulators and isolators to couple the signal back to the tester side of the link without providing an alternate downlink signal path. The DUT was placed on an azimuth positioning turntable in the center of the test volume as shown in the photos of Figure 7. A laptop was located nearby to serve as the client side for performing throughput measurements between the tester and DUT.
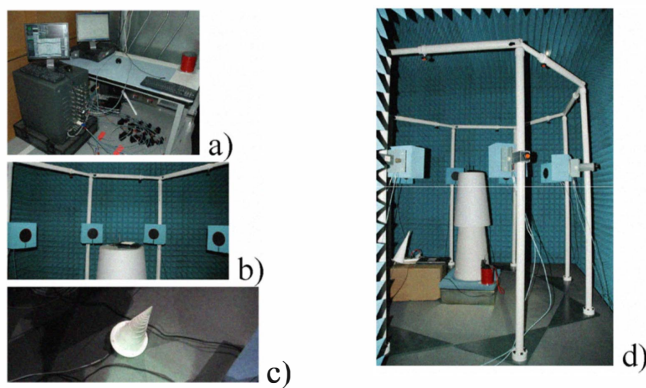

Fig 7. Photos of the experimental test set-up: a) the output of channel emulator is connected to 8 power amplifiers (Mini-Circuit ZVE-8G), b) DUT (access point of IEEE802.11n) in the center of the chamber, c) return link directed through conical log spiral antenna, and d) dual polarized OTA antennas surrounding the DUT mounted on small azimuth positioner.

Experiments were performed using 3 different types of channel models characterized in Table 1. The first one was modified 3GPP SCM Urban Micro model, where the delays were squeezed to zero while keeping the original angular behavior. Next, a modified TGn-C model, consisting of the delay profile from $\mathrm{TGn}-\mathrm{C}$, but the angular behavior from the SCM Urban Micro model was developed, followed by another modified TGn-C model having low correlation (10 wavelength separation of $\mathrm{Tx}$ antennas) on the transmitter side. The following figure depicts the modified TGn-C models. 

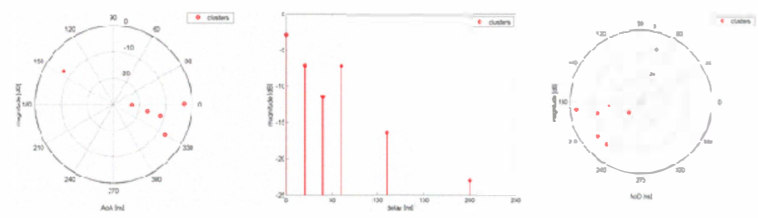

Fig 8. Graphical representation of the modified TGn-C models.

TABLE 1. CHANNEL MODEL CHARACTERISTICS

\begin{tabular}{|l|l|l|l|l|}
\hline Model & $\begin{array}{l}\text { Delay } \\
\text { spread } \\
{[\mathrm{ns}]}\end{array}$ & $\begin{array}{l}\text { Arrival } \\
\text { azimuth } \\
\text { spread }\left[^{\circ}\right]\end{array}$ & $\begin{array}{l}\text { Departure } \\
\text { azimuth } \\
\left.\text { spread }{ }^{\circ}\right]\end{array}$ & $\begin{array}{l}\text { TX } \\
\text { antenna } \\
\text { separation } \\
{[\lambda]}\end{array}$ \\
\hline $\begin{array}{l}\text { Modified } \\
\text { SCM } \\
\text { Urban } \\
\text { Micro }\end{array}$ & 0 & 68 & 18 & 0.3 \\
\hline $\begin{array}{l}\text { Modified } \\
\text { TGn-C }\end{array}$ & 30 & 68 & 18 & 0.3 \\
\hline $\begin{array}{l}\text { Modified } \\
\text { TGn-C } \\
\text { (low } \\
\text { correlation) }\end{array}$ & 30 & 68 & 18 & 10 \\
\hline
\end{tabular}

Figure 9 depicts the throughput vs. attenuation (intervening path loss) results as a function of rotating the DUT on the turntable in 30 degree increments using the modified TGn-C low correlation model. The goal is to evaluate the effect of altering the relative antenna orientation within the simulated environment for the selected channel model.

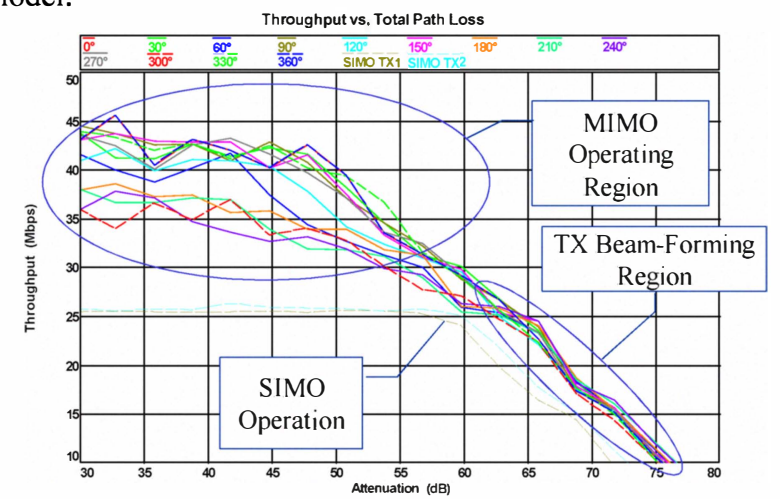

Figure 9. Throughput vs. Attenuation azimuth cut for the low correlation TGn-C model illustrating different regions of operation and comparing to SIMO behavior for same DUT.

We see that the MIMO throughput performance varies by as much as 10 Mbps ( 25\%) depending on the antenna orientation. Both the angular dependent throughput at a given signal level (attenuation setting) and/or the average throughput for all angles (Figure 10) are potential metrics for evaluating MIMO DUT performance. Such a metric measured at a single power level and reported only as an average of all angles has the advantage of being a relatively quick test. Conversely, looking at the total path loss, which is proportional to received power at the DUT, at a fixed throughput of 30 Mbps shows an angular dependent variation of about $10 \mathrm{~dB}$. This metric represents a "MIMO Sensitivity", where the signal level is lowered to determine the point where throughput falls below an arbitrary target throughput rate, as opposed to a target error rate.

It is clearly seen that there are at least two modes of MIMO operation in this device. The curves at the low attenuation end are clustered to $35-40 \mathrm{Mbps}$ and to $40-45$ Mbps, indicating some level of fall-back between at least two different modulation and coding schemes (MCS) within the $2 \times 2$ MIMO specification of $802.11 \mathrm{n}$. As the attenuation increases, the MIMO performance converges to performance that is independent of the DUT orientation due to the symmetry of the sleeve dipole elements. The same "waterfall" curve of the lower 802.11 data rates seen in the SIMO test cases is evident but with a $3 \mathrm{~dB}$ shift indicating the likely presence of transmit beam forming in the test system.

As we change the channel model to high correlation TGn$\mathrm{C}$ model, the throughput behaves as follows.

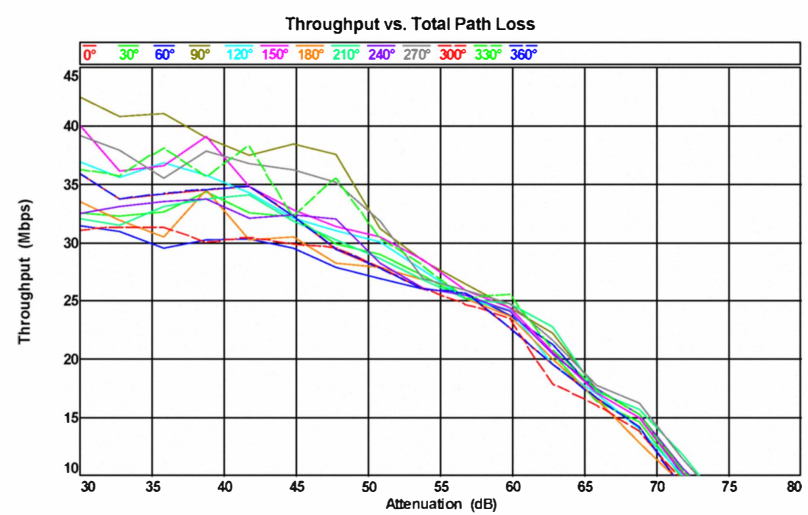

Figure 10. Throughput vs. Attenuation azimuth cut for the high correlation modified TGn-C model.

As seen, comparing to Figure 9, the throughput values in MIMO mode (i.e. low attenuation) are around 5 Mbps lower (see Figure 9 for a comparison of the average throughput). This is well understood, since the MIMO operates best in low correlation case.

Figure 11 shows the results of using the modified SCM Urban Micro Model. The results are quite similar to that of the high correlation TGn-C model. 


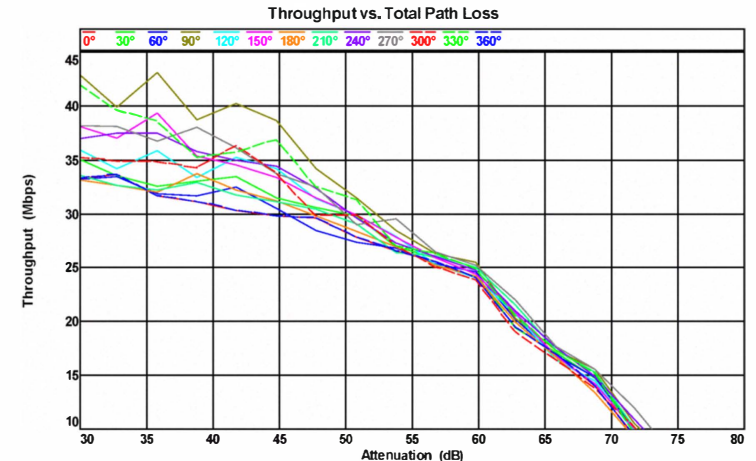

Figure 12. Throughput vs. Attenuation azimuth cut for the modified SCM Urban Micro model.

\section{ON TDD MIMO OTA TEST SET-UP}

The raising interest on Time division duplex (TDD) systems will be addressed in this chapter. TDD can be measured, as shown in previous chapter (IEEE802.11n is TDD), in downlink direction. However, the TD-LTE type systems use extensively the uplink information to characterize downlink. Also, typically more than two TX antennae is used.

From the radio channel modelling perspective, the same mapping algorithm will apply, as we are only mapping the local environment of the terminal.

From technical perspective, we need to pay attention to guarantee the link budget in both directions. One possible implementation of TDD MIMO OTA is shown below.

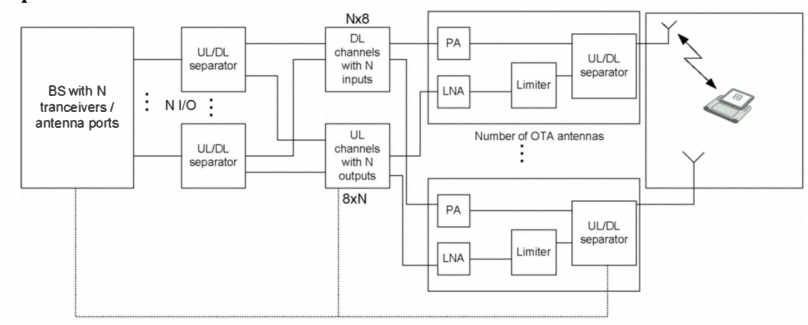

Figure 12. MIMO OTA TDD implementation.

\section{Conclusion}

This paper presented the MIMO OTA test system, which is meant for testing compact antenna devices. The paradigm of testing is changing due the fact that MIMO performance is defined by the radio channel characteristics and antenna characteristics. Thus the test system should consider both at the same time. The models we need to use are always geometry based stochastic models as the correlation properties are obtained by the angular behavior.

In this paper we discussed the experimental investigations of MIMO OTA system done with commercial, off-the-shelf IEEE802.11n device. The MIMO OTA system is capable of repeating MIMO performance in the laboratory conditions.

We showed through measurements that throughput is highly dependent on the channel model we are using, thus highlighting the fact that in order to have realistic results, the channel model should be appropriately selected. The MIMO OTA itself is most suitable for geometrical models, such as SCM, SCME and WINNER type of models.

We showed through measurements that MIMO performance is dependent on antenna orientation, thus angular information plays extremely important role when assessing the MIMO performance.

\section{ACKNOWLEDGMENT}

Part of this work has been performed in the framework of the CELTIC project CP5-026 WINNER+. The authors would like to acknowledge the contributions of their colleagues to the project.

\section{REFERENCES}

[1] M. D. Foegelle,"Over-the-air performance testing of wireless devices with multiple antennas", RF Design, Feb 2006, pp. $44-52$.

[2] P. Kyösti et. Al., "OTA testing for multiantenna terminals", TD (08) 670, COST2100 action, Lille, France, 6th MCM, 2008.

[3] J.-P. Nuutinen et. Al., "Experimental Investigations of OTA System", TD (09) 753, COST2100 action, Braunschweig, Germany, 7th MCM, 2009.

[4] P. Kyösti et. Al. "Proposal for standardized test procedure for OTA testing of multi-antenna terminals", TD (09) 766, COST2100 action, Braunschweig, Germany, 7th MCM, 2009.

[5] P. Kyösti et. Al., "Reconstruction and measurement of Spatial Channel Model for OTA" TD (09) 860, COST2100 action, Valencia, Spain, 8th MCM, 2009

[6] P. Kyösti et. Al., "Requirements for Channel Models for OTA Multiantenna Terminal Testing", TD (09) 859, COST2100 action, Valencia, Spain, 8th MCM, 2009.

[7] A. Scannavini et. Al., " Practical Considerations on MIMO OTA testing", TD (09) 854, COST2100 action, Valencia, Spain, 8th MCM, 2009.

[8] P. Kyösti et. Al., "Channel Modelling for Radiated Testing of MIMO Capable Terminals", Proceedings ICT Mobile Summit 2009, Santander, Spain, June 2009

[9] J-P. Nuutinen et. Al. " Effect of channel model simplification on throughput in MIMO OTA", TD(09)971, Vienna, Austria, COST2100 9th MCM, 2009

[10] J-P. Nuutinen et. Al., " Experimental Investigations of MIMO Performance of IEEE802.11n device in MIMO OTA test system", TD(09)972, Vienna, Austria, COST2100 9th MCM, 2009.

[11] T. Laitinen et. Al., "On the number of OTA antenna elements for plane-wave synthesis in a MIMO OTA test system involving circular antenna array", TD(09)976, Vienna, Austria, COST2100 9th MCM.

[12] P. Kyösti et. Al., " Verification of MIMO OTA set-up via simulations and measurements", $\operatorname{TD}(09) 990$, Vienna, Austria, COST2100 9th MCM, 2009.

[13] Elektrobit Website [Available online] http://www.elektrobit.com/what_we deliver/wireless_communicatio ns tools/list of publications/order eb propsim mimo ota white pa per

[14] 3GPP TR 25.996, "3rd Generation Partnership Project; technical specification group radio access networks; Spatial channel model for MIMO simulations (release 6)", V6.1.0.

[15] IST-WINNER II Deliverable 1.1.2 v.1.2, "WINNER II Channel Models", IST-WINNER2, Tech. Rep., 2008 (http://projects.celticinitiative. org/winner+/deliverables.html).

[16] P. Kyösti et. Al. " MIMO OTA test concept with experimental and simulated verification", To be published in EUCAP 2010, Barcelona, Spain, April 2010 